\title{
PREDICTING OUTCOMES OF NBA BASKETBALL GAMES
}

Eric Scot Jones and Rhonda C. Magel ${ }^{1 *}$

*1 Department of Statistics North Dakota State University Fargo, ND 58108

\section{*Corresponding Author: -}

\begin{abstract}
: -
Models are developed to help explain the point spread of an NBA basketball game based on significant in-game statistics. The models are based on a stratified random sample of 144 NBA basketball games played over a period of three years between 2008 and 2011. The in-game statistics found to be significant include the following: field goal shooting percentage; three-point shooting percentage, free throw shooting percentage, offensive rebounds, assists, turnovers, and free throws attempted. The models were validated using a random sample of 50 NBA games from the 2011-12 season. When these in-game statistics were known, the models were able to accurately tell which team had won the game between $88 \%$ and $94 \%$ of the time. The models were then used to make predictions using various methods to estimate the in-game statistics ahead of time. The models were used to predict winners of each game for the 2013, 2014, and the 2015 NBA Championships before any of the playoffs began. The models correctly predicted the overall champion each time. Predictions are also made for the 2016 NBA playoffs.
\end{abstract}

Keywords: - ordinary least squares regression, logistic regression, in-game statistics, seasonal averages 


\section{INTRODUCTION}

There are 30 teams in the National Basketball Association (NBA) and each team consists of 12 players. The starting lineup for a team is composed of five players playing in the following positions: point guard; shooting guard, small forward, power forward, and center. The remaining seven players are referred to as the secondary unit and serve as backup players. Only five players are allowed on the court at a time for each team.

The NBA is made up of two conferences (East and West) and each of the conferences have three divisions. The three divisions in the Eastern conference are the Atlantic, Central, and Southeast. The three divisions in the Western conference are Southwest, Northwest, and Pacific. The regular season begins in late October and goes through April with each of the 30 teams playing 82 games [1].

The NBA playoffs follow the regular season. The top eight teams in each of the conferences compete in the playoffs with the teams selected from each conference seeded as 1 (best) through 8 (worst). In the first round of the playoffs the teams in each conference whose seeds add up to 9 play each other. Each round of the playoffs is a best of seven series. The series are played in a 2-2-1-1-1 format with the top seeded team playing the first two games at home, followed by 2 away, and then alternating home and away if needed. The rounds include the $1^{\text {st }}$ round, conference semi-finals, conference finals, and NBA finals [1].

The four winners of the first round of each conference advance to the conference semi-finals with the winner of the 1-8 seeds playing the winner of the $4-5$ seeds, and the winner of the 2-7 seeds playing the winner of the 3-6 seeds. This process continues through the finals [1].

NBA basketball is popular. The NBA finals were the second most watched sporting even after the Super Bowl in 2015 according to the Nielson ratings [2]. Revenue for the NBA has also increased by 2.13 billion dollars annually since the 2001-02 season. The projected revenue for the 2014-15 season was 5.18 billion dollars [3].

Because of the popularity of the NBA and the money associated with it, we would like to develop models based on ingame statistics to help explain the final point spread of the game and also to estimate the probability of a team winning the game based on the in-game statistics. The developed models will give both fans and coaches an idea as to what ingame statistics their teams should concentrate on to win more games. We would also like to try and use the models to make future predictions on games by estimating the in-game statistics ahead of time before the game is played. Various method will be tried to estimate the in-game statistics.

There has been similar past research related to NCAA Division 1 Men's Basketball games. In particular, Magel and Unruh [4] developed a least squares regression model to help explain the point spread in an NCAA game based on in-game statistics. They considered eleven in-game statistics for their model and found the following four to be significant: difference in assists; difference in turnovers; difference in free throw attempts; and difference in defensive rebounds. Much research has also been done on predicting the results of March Madness each year such as that proposed in $[5,6,7,8]$. The major distinction between developing models to predict March Madness results and developing models to predict NBA playoff results is that games in March Madness are single elimination games so if a team loses one game, it is out of the tournament. Each match-up of two teams in the NBA tournament is a best of seven game series.

\section{Developing the Models}

A stratified random sample was used to collect data from the 30 NBA teams over 3 seasons 2008-09 through 2010-2011. A random number generator was used to sample 144 games in order to ensure that games were evenly dispersed throughout each of the seasons. Data was collected in terms of the reference team, referred to as "Team A", and the opposing team, referred to as "Team B". Differences were found between all of the ingame statistics between "Team A" and "Team B". The differences collected from each game are given in Table 1. All of the data come from box scores on the USA Today website from 2008-2011 [9]. The year in which the game was played was noted when data was collected.

\begin{tabular}{|l|l|l|}
\hline Table 1 - Variables for Team Comparisons \\
\hline Variable & Code Name & Description \\
\hline Point Spread & PSD & $\begin{array}{l}\text { The difference in total number of points scored between Team A and } \\
\text { Team B }\end{array}$ \\
\hline Field Goal Shooting & FGS & $\begin{array}{l}\text { The difference in field goal shooting percentage between Team A and } \\
\text { Team B }\end{array}$ \\
\hline Three Point Shooting & 3PS & $\begin{array}{l}\text { The difference in three-point shooting percentage between Team A } \\
\text { and Team B }\end{array}$ \\
\hline Free Throw Shooting & FTS & $\begin{array}{l}\text { The difference in the number of free throws made between Team A } \\
\text { and Team B }\end{array}$ \\
\hline Free Throws Made & FTM & $\begin{array}{l}\text { The difference in the number of free throws attempted between Team } \\
\text { A and Team B }\end{array}$ \\
\hline Free Throws Attempted & FTA & The difference in the number of assists between Team A and Team B \\
\hline Assists & AST & $\begin{array}{l}\text { The difference in the number of turnovers between Team A and Team } \\
\text { B }\end{array}$ \\
\hline Turnovers & TOS & $\begin{array}{l}\text { The difference in the number of offensive rebounds between Team A } \\
\text { and Team B }\end{array}$ \\
\hline Offensive Rebounds & OR & $\begin{array}{l}\text { The difference in the number of defensive rebounds between Team A } \\
\text { and Team B }\end{array}$ \\
\hline Defensive Rebounds & DR & $\begin{array}{l}\text { The difference in the number of total rebounds between Team A and } \\
\text { Team B }\end{array}$ \\
\hline Total Rebounds & TR &
\end{tabular}




\subsection{Point Spread Model}

The stepwise selection technique was used in developing an ordinary least squares regression model with point spread as the dependent variable. The remaining 10 variables given in Table 1 were considered for entry into the model using an $\alpha=0.15$ significance level for entry and exit into and out of the model. Seven variables were found to be significant in the model. These variables, along with their parameter estimates, and associated p-values are given in Table 2 . This model had an adjusted $\mathrm{R}^{2}$ value of 0.9145 , and thus approximately $91.45 \%$ of the variation in point spread of a basketball game was explained by the seven variables given in Table 2 .

\begin{tabular}{|l|l|l|l|l|l|}
\hline \multicolumn{6}{|l|}{ Table 2 - Parameter Estimates and T-tests for Point Spread Model } \\
\hline Source & DF & $\begin{array}{l}\text { Parameter } \\
\text { Estimate }\end{array}$ & $\begin{array}{l}\text { Standard } \\
\text { Error }\end{array}$ & t Value & P value \\
\hline FGS & 1 & 1.485 & .059 & 25.14 & $<.0001$ \\
\hline 3PS & 1 & .169 & .020 & 8.34 & $<.0001$ \\
\hline FTS & 1 & .196 & .025 & 7.80 & $<.0001$ \\
\hline OR & 1 & .879 & .062 & 14.07 & $<.0001$ \\
\hline AST & 1 & .239 & .067 & 3.55 & 0.0005 \\
\hline TOS & 1 & -.837 & .077 & -10.85 & $<.0001$ \\
\hline FTA & 1 & .337 & .037 & 8.99 & $<.0001$ \\
\hline
\end{tabular}

Indicator variables representing the different years that the basketball games were played were placed into the model and tested for significance. They were not found to be significant with $p$-value $>0.10$ indicating that the model developed should be good for any year. Residual analysis was conducted on the model and no violation of the error term assumptions was found [10]. The model is given in equation 1 .

$$
\text { PSD }=1.485(\text { FGS })+.169(3 P S)+.196(\text { FTS })+.879(\text { ORS })+.239(\text { ASTS })+\quad \text { (Eq. 1) }(-.837)(\text { TOS })+.337(\text { FTAS })
$$

It is noted that on average for every extra turnover a team has over another team, the point difference is approximately .84 points less. Every extra assist a team has increases the point spread by approximately 0.24 points over the opposing team

\subsection{Logistic Regression Model}

A logistic model was next developed to estimate the probability that "Team A" won the game based on ingame statistics found to be significant. The dependent variable in the model was equal to " 1 " if "Team A" won the game and " 0 " if "Team A" lost the game. The stepwise selection technique with an alpha equal to 0.15 for entry and exit was used to help determine the in-game statistics that were significant in the model. The same 7 in-game statistics that were found to be significant in the point spread model were found to be significant in the logistic model. The parameter estimates and associated p-values for each of the predictors are given in Table 3. Indicator variables for the year the game was placed were placed in the model, tested for significance, and found not to be significant ( $\mathrm{p}$-value $>0.10$ ). This last result indicates that the model developed should be good for any year. The model is given in equation 2 .

$\exp [.985(\mathrm{FGS})+.168(3 \mathrm{PS})+.146(\mathrm{FTS})+.517(\mathrm{ORS})+.276(\mathrm{AST})+(-.661)(\mathrm{TOS})+.410(\mathrm{FTA})] /(1+\exp [.985(\mathrm{FGS})$ $+.168(3 \mathrm{PS})+.146(\mathrm{FTS})+.517(\mathrm{ORS})+.276(\mathrm{AST})+(-.661)(\mathrm{TOS})+.410($ FTA $)])$ (Eq.2)

\begin{tabular}{|l|l|l|l|l|}
\hline \multicolumn{5}{|l|}{ Table 3- Parameter Estimates and Chi-Square -tests for Logistic Model } \\
\hline $\begin{array}{l}\text { Predictor } \\
\text { Constant }\end{array}$ & Coefficient & $\begin{array}{l}\text { Standard Error } \\
\text { Coefficient }\end{array}$ & Chi - Square & P Value \\
\hline FGS & .985 & .253 & 69.98 & 0.000 \\
\hline 3PS & .168 & .0561 & 27.62 & 0.000 \\
\hline FTS & .146 & .0551 & 14.37 & 0.000 \\
\hline OR & .517 & .147 & 30.12 & 0.000 \\
\hline AST & .276 & .104 & 9.59 & 0.002 \\
\hline TOS & -.661 & .215 & 24.01 & 0.000 \\
\hline FTA & .410 & .121 & 44.98 & 0.000 \\
\hline
\end{tabular}

\section{Validating the Models}

Fifty games were sampled from the 2011-2012 NBA season to validate the point spread model. This was done by randomly selecting one of the six divisions and then randomly sampling 10 games from each of the five teams in that division. None of these games was used in the development of the models. The in-game statistics were collected from these games from the USA Today box scores [9]. The actual differences between the significant in-game statistics were placed into the model using Eq. 1. If the estimated point spread was positive, it was predicted that "Team A" won the game, and if the estimated point spread was negative, it was predicted that "Team A" lost the game.

Table 4 gives an example as to how the model was validated. The data given are the in-game statistics from a game played between the Phoenix Suns (Team A) and the Los Angeles Lakers (Team B) on January 10, 2012. 


\begin{tabular}{|l|l|l|l|}
\hline \multicolumn{3}{|l|}{ Table 4 - Data Collection Example for Point Spread Model Validation } \\
\hline Variable & $\begin{array}{l}\text { Phoenix Suns } \\
\text { "Team A" }\end{array}$ & $\begin{array}{l}\text { Los Angeles Lakers } \\
\text { "Team B" }\end{array}$ & Difference \\
\hline FGS (\%) & 42.5 & 48.8 & -6.3 \\
\hline FTS (\%) & 66.7 & 82.6 & -15.9 \\
\hline 3PS (\%) & 35.0 & 11.8 & 23.2 \\
\hline OR & 9 & 14 & -5 \\
\hline FTA & 12 & 23 & -11 \\
\hline TOS & 11 & 14 & -3 \\
\hline AST & 18 & 27 & -9 \\
\hline
\end{tabular}

The values for the difference between "Team A" - "Team B" were then entered into (Eq. 1) to obtain the estimated point spread.

Point Spread $=1.485(-6.3)+0.169(23.2)+0.195(-15.9)+0.879(-5)+0.337(-11)+0.239(-9)-0.837(-3)=-16.27$

Since the point spread is negative, a loss was predicted for the Phoenix Suns ("Team A").

There were 47 games correctly predicted out of 50 for an accuracy of $94 \%$. The results are given in Table 5 .

\begin{tabular}{|l|l|l|l|l|}
\hline \multicolumn{2}{|l|}{ Table 5- Point Spread Model Validation Summary } \\
\hline PSD Model & \multicolumn{2}{|l|}{ Actual } & Total \\
\cline { 3 - 5 } & Win & 1 & 15 \\
\hline Predicted & Win & 14 & 33 & 35 \\
\cline { 2 - 5 } & Lose & 2 & & \\
\hline
\end{tabular}

The same data was used to validate the logistic regression model. The differences between the significant ingame statistics were placed in the models. If the logistic model estimated the probability that Team A would win was higher than 0.5 , we predicted Team A would win. Otherwise, it was predicted that Team A would lose. As an example as to how this validation process worked, consider the in-game statistics between the Suns and the Lakers for the game played on January 10, 2012, given in Table 4. The estimated probability for the Suns

("Team A") to win was

$\exp [.985(-6.3)+.168(23.2)+.146(-15.9)+.517(-5)+.276(-9)+(-.661)(-3)+.410(-11)] / 1+\exp [.985(-6.3)+.168(23.2)$

$+.146(-15.9)+.517(-5)+.276(-9)+(-.661)(-3)+.410(\mathrm{~F}-11)]=.00005$

Therefore, it was predicted that the Suns ("Team A") would lose this game. This process was done for all 50 games in the sample. The logistic model correctly predicted 44 out of 50 games for an accuracy of $88 \%$. A summary of the results is given in Table 6 .

\begin{tabular}{|l|l|l|l|l|}
\hline \multicolumn{5}{|l|}{ Table 6- Logistic Model Validation Summary } \\
\hline \multirow{2}{*}{ Logistic Model } & Actual \\
\cline { 3 - 5 } \multicolumn{2}{|c|}{} & Win & Lose & Total \\
\hline \multirow{3}{*}{ Predicted } & Win & 12 & 2 & 14 \\
\cline { 2 - 5 } & Lose & 4 & 32 & 36 \\
\cline { 2 - 5 } & Total & 16 & 34 & 50 \\
\hline
\end{tabular}

\section{Using the Models for Predictions}

The point spread and logistic regression models were next used to make predictions for future games by estimating the in-game statistics found to be significant ahead of time. There were initially three different methods implemented for estimating these in-game statistics. These methods included using a 3-game moving average, a 3-game moving median, and a 3-game moving weighted average.

In order to predict a winner of a particular game between "Team A" and "Team B", we considered the three previous games played by "Team A" and the three previous games played by "Team B". The average of the ingame statistics for "Team A" from the three previous games was calculated, the median of the in-game statistics for "Team A" from the three previous games was also calculated, an a weighted average based on the in-game statistics for "Team A" of the three previous games was found for each statistic by multiplying the median value of the three by 2 , the smallest and largest value by 1 and then dividing by 4 . The same process was followed using the three previous games played by "Team B". 
The differences between "Team A" and "Team B" were found for the mean, median, and weighted average of each of these in-game statistics and placed into the model.

The point spread or probability that "Team A" would win was estimated.

The point spread model correctly predicted 375 out of 604 games correctly for an accuracy of $62 \%$ when the 3 -game moving average was used to estimate the in-game statistics. It correctly predicted 344 out of 604 games for an accuracy of $57 \%$ when the 3-game moving median was used, and it correctly predicted 362 out of 604 games correctly for an accuracy of $60 \%$ when the weighted average was used.

The logistic model correctly predicted 365 out of 604 games correctly for an accuracy of $60 \%$ when the 3 game moving average was used in place of the in-game statistics. It correctly predicted 326 out of 604 games correctly for an accuracy of $54 \%$ when using the 3-game moving median, and 344 out of 604 correctly for an accuracy of $57 \%$ when using the 3game weighted average.

\section{Using the Point Spread Model With 3-game Moving Average to Predict the 2013 NBA Playoffs}

The point spread model using the 3-game moving average was used to predict the 2013 NBA playoffs since the point spread model appeared to do slightly better than the logistic model and the 3-game moving average appeared to do slightly better than the other two techniques when estimating the in-game statistics. The first round of the NBA playoffs in the Eastern Conference had the following match-ups: Miami Heat vs Milwaukee Bucks; New York Knicks vs Boston Celtics; Indiana Pacers vs Atlanta Hawks, and Brooklyn Nets vs Chicago Bulls. The first round in the Western Conference had the following match-ups: Oklahoma City Thunder vs Houston Rockets, San Antonio Spurs vs Los Angeles Lakers, Denver Nuggets vs Golder State Warriors, and Los Angeles Clippers vs Memphis Grizzlies.

Using the point spread model with the 3-game moving average, the predicted winners of the $1^{\text {st }}$ round in the Eastern Conference were Miami Heat, New York Knicks, Atlanta Hawks, and Brooklyn Nets. The teams that actually advanced to the second round of the Eastern Conference playoffs were Miami Heat, New York Knicks, Indiana Pacers, and Chicago Bulls, giving the model a 50\% accuracy. The predicted winners of the $1^{\text {st }}$ round in the Western Conference were Oklahoma City Thunder, San Antonio Spurs, Denver Nuggets, and Los Angeles Clippers. The actual winners were Oklahoma City Thunder, San Antonio Spurs, Golden State Warriors, and Memphis Grizzlies giving the model 50\% accuracy for the $1^{\text {st }}$ round in the Western Conference also.

The model predicted that for the second round in the East, the winners would be Miami Heat and the New York Knicks. The actual winners were the Miami Heat and Indiana Pacers, for an accuracy of 50\%. The predicted second round winners in the West were Oklahoma City Thunder and San Antonio Spurs with the actual winners being the Memphis Grizzlies, and the San Antonio Spurs, for an accuracy of $50 \%$.

The model predicted that the Eastern conference final winner would be the Miami Heat who did actually win. However, the model predicted that the Western conference winner would be the Oklahoma City Thunder, but actually San Antonio won. The model again had an accuracy of $50 \%$ in this round.

Overall, the model predicted that Miami Heat would win the NBA championship and Miami Heat actually did win. In summary, the model predicted 8 out of 15 match-ups correctly, for an accuracy of $53 \%$.

\section{Using Seasonal Averages to Replace In-Game Statistics}

Overall, we found that the 3-game moving average did not work well in estimating in-game statistics for the NBA playoffs. We decided to try seasonal averages instead. During the 2013-14 season we randomly selected 62 games in which to try to make predictions ahead of time. For these 62 games, seasonal averages calculated up to just before the game was played were used to estimate the significant in-game statistics. Fortyfour of the 62 games selected were accurately predicted by the point spread model for an overall accuracy of $70.9 \%$.

\section{Predicting 2014 and 2015 NBA Playoffs}

In predicting 2014 NBA playoffs, we used the point spread model with seasonal averages used in place of the in-game statistics to make predictions as to which team would win. We decided to compare how well this method did with just calculating the difference in the seasonal average point differential between the two teams playing in each round of the playoffs. We also tried the method of using a weighted average between the point spread estimate obtained from the model and the difference in the seasonal average point differential between the two teams playing in the playoffs. For this weighted average, we took 0.70 times the estimated point spread obtained from the point spread model plus 0.30 times the difference in the average point differential between the two teams playing in the playoff round.

Prior to the start of the 2014 NBA playoffs, seasonal averages were collected for each of the 16 teams competing. Predictions for each round of the playoffs were then made using each of the three methods before any game in the playoffs had started. As an example as to how predictions were made, the Wizards and Bulls were to play each other in round one of the 2014 NBA playoffs. The seasonal averages for both the Wizards and the Bulls of all the significant in-game statistics in the model are given in Table 7. When these differences were placed into the point spread model, the following was obtained:

Point Spread $=1.485(2.7)+0.169(3.2)+0.195(-4.8)+0.879(-.6)+0.337(-2.4)+0.239(.6)-0.837(-.2)=2.5889$

Based on the calculation, a win is predicted for the Washington Wizards.

Using the average points differential: $1.3-1.9=-0.6$, which predicts a loss for the Washington Wizards.

Using the weighted model: $2.5889(.7)+-.6(.3)=1.6322$, which predicts a win for the Washington Wizards. It is noted that two methods give us a positive point spread and one method a negative point spread. Anytime a discrepancy was observed in the different methods for predicting the point spread, the weighted model was used. Based on all of these 
predictions, the Washington Wizards would be expected to win more games in the best of 7 series. Round 1 winners were predicted and the weighted point spread model was then used to predict winners of round 2 based on the predicted matchups from the model. This process continued until the 2014 NBA champion was predicted. In some cases, the predicted match-ups were different than the actual match-ups.

\begin{tabular}{|l|l|l|l|}
\hline Table 7 - Regular Seasonal Averages (Wizards vs Bulls) \\
\hline Variable & $\begin{array}{l}\text { Washington Wizards } \\
\text { "Team A" }\end{array}$ & $\begin{array}{l}\text { Chicago Bulls } \\
\text { "Team B" }\end{array}$ & Difference \\
\hline FGS (\%) & 45.9 & 43.2 & 2.7 \\
\hline FTS (\%) & 73.1 & & -4.8 \\
\hline 3PS (\%) & 38.0 & 34.8 & 3.2 \\
\hline ORS & 10.8 & 11.4 & -0.6 \\
\hline FTA & 20.9 & 23.3 & -2.4 \\
\hline TOS & 14.7 & 14.9 & -0.2 \\
\hline AST & 23.3 & 22.7 & 0.6 \\
\hline Points Differential & 1.3 & 1.9 & -0.6 \\
\hline
\end{tabular}

Figure 1 gives the predicted 2014 NBA playoffs Eastern Conference Outcome and Figure 2 gives the actual 2014 Eastern Conference Outcome. Figure 3 gives the predicted 2014 NBA playoffs Western Conference Outcome and Figure 4 gives the actual 2014 Western Conference Outcome. Figures 5 and 6 give the predicted 2014 NBA final results and the actual results, respectively. The correct two teams were predicted to be in the finals and the winner was correctly predicted.

Using this process with the weighted model, we correctly predicted 12 out of 15 games correctly, for an accuracy of $80 \%$ correct. If just the point spread model was used, it would have predicted only 9 out of the 15 games correctly for an accuracy of $60 \%$. If the difference in the average points differential was used by itself, it would have predicted 11 out of 15 games correctly for an accuracy of $73 \%$. The weighted model did the best in this case.

\begin{tabular}{|l|l|l|l|}
\hline PACERS & \multirow{2}{*}{ PACERS } & \\
\cline { 1 - 1 } HAWKS & \multirow{2}{*}{ WIZARDS } & \\
\cline { 1 - 1 } BULLS & WIZARDS & & \multirow{2}{*}{ HEAT } \\
\cline { 1 - 1 } WIZARDS & RAPTORS & & \\
\cline { 1 - 1 } RAPTORS & HEAT & \\
\cline { 1 - 1 } NETS & HEAT & & \\
\cline { 1 - 2 } HEAT & & \\
\hline
\end{tabular}

Figure 1 - Predicted 2014 NBA Playoffs Eastern

\begin{tabular}{|c|c|c|c|}
\hline PACERS & & \multirow{4}{*}{ *PACERS } & \multirow{8}{*}{ HEAT } \\
\hline HAWKS & PACERS & & \\
\hline BULLS & \multirow[b]{2}{*}{ WIZARDS } & & \\
\hline WIZARDS & & & \\
\hline RAPTORS & \multirow[b]{2}{*}{ *NETS } & \multirow{4}{*}{ HEAT } & \\
\hline NETS & & & \\
\hline HEAT & \multirow[b]{2}{*}{ HEAT } & & \\
\hline BOBCATS & & & \\
\hline
\end{tabular}

Figure 2 - Actual 2014 NBA Playoffs Eastern Conference Outcome* When actual differs from predicted 


\begin{tabular}{|c|c|c|c|}
\hline SPURS & & \multirow{4}{*}{ SPURS } & \multirow{8}{*}{ SPURS } \\
\hline MAVERICKS & SPURS & & \\
\hline ROCKETS & \multirow[b]{2}{*}{ ROCKETS } & & \\
\hline BLAZERS & & & \\
\hline CLIPPERS & \multirow[b]{2}{*}{ CLIPPERS } & \multirow{4}{*}{ THUNDER } & \\
\hline WARRIORS & & & \\
\hline THUNDER & \multirow[b]{2}{*}{ THUNDER } & & \\
\hline GRIZZLIES & & & \\
\hline
\end{tabular}

Figure 3 - Predicted 2014 NBA Playoffs Western Conference Outcome

\begin{tabular}{|l|l|l|l|}
\hline SPURS & \multirow{2}{*}{ SPURS } & \multirow{2}{*}{ SPURS } & \\
\cline { 1 - 2 } MAVERICKS & \multirow{2}{*}{ *BLAZERS } & & \multirow{2}{*}{ SPURS } \\
\cline { 1 - 2 } ROCKETS & CLIPPERS & \multirow{2}{*}{ THUNDER } & \\
\cline { 1 - 2 } BLAZERS & THUNDER & & \\
\cline { 1 - 2 } CLIPPERS & & \\
\cline { 1 - 2 } THARRIORS & & \\
\cline { 1 - 2 } GRIZZLIES & &
\end{tabular}

Figure 4 - Actual 2014 NBA Playoffs Western Conference Outcome * When actual differ from predicated.

\begin{tabular}{|l|l}
\hline SPURS & \\
\cline { 1 - 1 } HEAT & SPURS \\
\hline
\end{tabular}

Figure 5 - Predicted 2014 NBA Finals

\begin{tabular}{|l|l}
\hline SPURS & \\
\cline { 1 - 1 } HEAT & SPURS \\
\end{tabular}

Figure 6 - Actual 2014 NBA Finals

The actual process was repeated for the 2015 NBA Playoffs. The results for the predicted outcomes and actual outcomes are shown in Figures 7-12.

\begin{tabular}{|c|c|c|c|}
\hline HAWKS & & \multirow{4}{*}{ HAWKS } & \multirow{8}{*}{ CAVALIERS } \\
\hline NETS & HAWKS & & \\
\hline RAPTORS & \multirow[b]{2}{*}{ *WIZARDS } & & \\
\hline WIZARDS & & & \\
\hline BULLS & & \multirow{4}{*}{ CAVALIERS } & \\
\hline BUCKS & *BULLS & & \\
\hline CAVALIERS & \multirow[b]{2}{*}{ CAVALIERS } & & \\
\hline CELTICS & & & \\
\hline
\end{tabular}

Figure 7 - Predicted 2015 NBA Playoffs Eastern Conference Outcome 


\begin{tabular}{|c|c|c|c|}
\hline HAWKS & & \multirow{4}{*}{ HAWKS } & \multirow{8}{*}{ CAVALIERS } \\
\hline NETS & HAWKS & & \\
\hline RAPTORS & \multirow[b]{2}{*}{ *WIZARDS } & & \\
\hline WIZARDS & & & \\
\hline BULLS & \multirow[b]{2}{*}{ *BULLS } & \multirow{4}{*}{ CAVALIERS } & \\
\hline BUCKS & & & \\
\hline CAVALIERS & \multirow[b]{2}{*}{ CAVALIERS } & & \\
\hline CELTICS & & & \\
\hline
\end{tabular}

Figure 8 - Actual 2015 NBA Playoffs Eastern Conference Outcome - * When actual differs from predicted

\begin{tabular}{|c|c|c|c|}
\hline WARRIORS & & \multirow{4}{*}{ WARRIORS } & \multirow{8}{*}{ WARRIORS } \\
\hline PELICANS & WARRIORS & & \\
\hline BLAZERS & \multirow[b]{2}{*}{ GRIZZLIES } & & \\
\hline GRIZZLIES & & & \\
\hline CLIPPERS & & \multirow{4}{*}{ CLIPPERS } & \\
\hline SPURS & CLIPPERS & & \\
\hline ROCKETS & \multirow[b]{2}{*}{ MAVERICKS } & & \\
\hline MAVERICKS & & & \\
\hline
\end{tabular}

Figure 9 - Predicted 2015 NBA Playoffs Western Conference Outcome

\begin{tabular}{|c|c|c|c|}
\hline WARRIORS & & \multirow{4}{*}{ WARRIORS } & \multirow{8}{*}{ WARRIORS } \\
\hline PELICANS & WARRIORS & & \\
\hline BLAZERS & \multirow[b]{2}{*}{ GRIZZLIES } & & \\
\hline GRIZZLIES & & & \\
\hline CLIPPERS & \multirow[b]{2}{*}{ CLIPPERS } & \multirow{4}{*}{ *ROCKETS } & \\
\hline SPURS & & & \\
\hline ROCKETS & \multirow[b]{2}{*}{ *ROCKETS } & & \\
\hline MAVERICKS & & & \\
\hline
\end{tabular}

Figure 10 - Actual 2015 NBA Playoffs Western Conference Outcome * When actual differs from predicted

\begin{tabular}{|l|l|}
\hline \multicolumn{1}{|c|}{ WARRIORS } & \multirow{2}{*}{ WARRIORS } \\
\hline CAVALIERS & \multirow{2}{|c|}{ Figure 11 - Predicted 2015 NBA Finals } \\
\hline WARRIORS & WARRRIORS \\
\hline CAVALIERS
\end{tabular}

Figure 12 - Actual 2015 NBA Finals

The process for 2015 using the weighted model correctly predicted 11 out of 15 for an accuracy of $73 \%$. When using the point spread model only, it correctly predicted 9 out of 15 games correctly, for an accuracy of $60 \%$. When the difference in the average points differential was used, it correctly predicted 10 out of 15 games correctly, for an accuracy of $67 \%$. The weighted model did the best in both years.

\section{Predicting the 2016 NBA Playoffs.}

The same process was used once again to predict the 2016 NBA playoffs before the playoffs began. The results of the predicted outcomes using the weighted model are shown in Figures 13-15. 


\begin{tabular}{|c|c|c|c|}
\hline CAVALIERS & & \multirow{4}{*}{ CAVALIERS } & \multirow{8}{*}{ CAVALIERS } \\
\hline PISTONS & CAVALIERS & & \\
\hline HAWKS & \multirow[b]{2}{*}{ HAWKS } & & \\
\hline CELTICS & & & \\
\hline HEAT & & \multirow{4}{*}{ RAPTORS } & \\
\hline HORNETS & HEAT & & \\
\hline RAPTORS & \multirow[b]{2}{*}{ RAPTORS } & & \\
\hline PACERS & & & \\
\hline
\end{tabular}

Figure 13 - Predicted 2016 NBA Playoffs Eastern Conference Outcome

\begin{tabular}{|c|c|c|c|}
\hline WARRIORS & & \multirow{4}{*}{ WARRIORS } & \multirow{8}{*}{ WARRIORS } \\
\hline ROCKETS & WARRIORS & & \\
\hline CLIPPERS & \multirow[b]{2}{*}{ CLIPPERS } & & \\
\hline BLAZERS & & & \\
\hline THUNDER & \multirow[b]{2}{*}{ THUNDER } & \multirow{4}{*}{ SPURS } & \\
\hline MAVERICKS & & & \\
\hline SPURS & \multirow{2}{*}{ SPURS } & & \\
\hline GRIZZLIES & & & \\
\hline
\end{tabular}

Figure 14 - Predicted 2016 NBA Playoffs Western Conference Outcome

\begin{tabular}{|l|l|}
\hline WARRIORS & \multirow{2}{*}{ WARRIORS } \\
\hline CAVALIERS & WARRIS \\
\hline
\end{tabular}

Figure 15 - Predicted 2016 NBA Finals

The NBA playoffs are presently still going on. We are currently in the conference finals. Out of 12 matches played so, far, the process has correctly predicted 10 of the match-ups for an accuracy of $83 \%$.

\section{Conclusions}

A model was developed to explain the point spread of a basketball game when the differences between two teams in the following in-game statistics were known: field goal shooting percentage; 3-point shooting percentage; free-throw shooting percentage; number of offensive rebounds; number of assists, number of turnovers, and number of free-throws attempted. This model explained $91.45 \%$ of the variation in the point spread when these in-game statistics were known. A logistic model was also developed to estimate the probability of a team winning the basketball game when in-game statistics were known. The logistic model found the same seven in-game statistics to be significant. Both of the models were validated using a random sample of 50 NBA games not used in the development of the models. The point spread model correctly predicted the winner $94 \%$ of the time when the seven in-game statistics were given. The logistic model correctly predicted the winner $88 \%$ of the time.

Both the point spread model and the logistic model were used to try and predict future basketball games. In this case, the seven in-game statistics needed to be estimated ahead of time. Three methods for estimating these statistics were tried including a 3-game moving average, a 3-game moving median, and a 3-game moving weighted average. It was determined from a sample of 604 games that the point spread model using the 3 -game moving average appeared to be doing the best, although the accuracy of this model was only $62 \%$. The 2013 NBA playoffs were predicted using the point spread model replacing the in-game statistics with the 3-game moving average and 8 out of the 15 match-ups were correctly predicted for an accuracy of $53 \%$. This was not that great, but the model did predict the correct champion.

Seasonal averages up to the point when a game was played were next tried in place of the in-game statistics to predict the winners in a sample of 62 games in the 2013-14 regular season. The point spread model correctly predicted the outcome of $70.9 \%$ of these games. It was decided that seasonal averages were better than using the 3-point moving averages to estimate the in-game statistics in the point spread model and they were easier to obtain.

Three methods were tried to predict the outcomes of both the 2014 and 2015 NBA playoffs. One method was to use the point spread model in equation 1 substituting seasonal averages in place on in-game statistics to make predictions on match-ups. The second method was to use the difference in the average point spread differential between the two teams playing in a match-up. The third method used a weighted average with $70 \%$ of the weight coming from predicted point spread from the model obtained in equation 1 using seasonal averages and $30 \%$ of the weight coming from the difference 
in the average points differential between the two teams. The third method did the best in predicting the outcomes for both the 2014 and 2015 playoffs. In 2014, 80\% of the match-ups were correctly predicted and in 2015 , 73\% of the matchups were correctly predicted. In both years, the two teams playing in the championship game and the actual champion team were correctly predicted. Predictions for the 2016 NBA playoffs were also made. Through the first 12 match-ups of the 2016 playoffs, the third method has correctly predicted 10 for an accuracy of $83 \%$. It appears this method is doing well at predicting the NBA playoff outcomes.

\section{REFERENCES}

[1].“2013-2014 NBA Schedule”. (2014). NBA.com: Turner Sports Digital. Retrieved from http://www.nba.com

[2].Tack, T. (2015). "NBA: A Model for Growth in the $21^{\text {st }}$ Century." Retrieved from http://sports.politicususa.com/2015/06/04/nba-a-model-for-growth-in-the-21st-century.html

[3]."NBA Player Salaries". (2016). ESPN.com: Retrieved from http://espn.go.com/nba/salaries [4] Magel, R. \& Unruh, S. (2013). Determining Factors Influencing the Outcome of College Basketball Games. Open Journal of Statistics, Vol.3, No. 4, August 2013.

[4].West, B.T. (2006). A Simple and Flexible Rating Method for Predicting Success in the NCAA Basketball Tournament. Journal of Quantitative Analysis in Sports, 2, 3, p 3-8.

[5].West, B.T. (2008). A Simple and Flexible Rating Method for Predicting Success in the NCAA Basketball Tournament: Updated Results from 2007. Journal of Quantitative Analysis in Sports, 4, 2, p 6-8.

[6].Pomeroy, K. (2014) Pomeroy's ratings. Available: http://www.kenpom.com.

[7].Shen, G., Hua, S., Zhang, S., Mu, Y., \& Magel, R. (2014). Predicting Results of March Madness Using the Probability Self-Consistent Method. International Journal of Sports Science. 5.4: 139-144

[8].“ NBA Schedule”. (2013). USA Today: A Garnnett Company. Retrieved from http://www.usatoday.com

[9].Abraham, B., and Ledolter, J. (2006). Introduction to Regression Modeling, Thomson Brooks/Cole Belmont, Ca. 